\title{
Robinsonnades dans l'île de la page : le soi face à l'isolement dans la littérature îlienne européenne après 1945
}

\author{
Ioana Andreescu \\ École des hautes études en sciences sociales (EHESS)
}

Le topos littéraire de lîle déserte se constitue en un genre littéraire bien présent dans la période de la reconstruction européenne qui suit la Seconde Guerre mondiale puisque l'espace insulaire offre d'excellentes possibilités de réflexion sur le système social, l'organisation politique, ainsi que sur l'évolution intérieure subie par les héros qui habitent l'île. Dans le nouveau contexte sociopolitique, les Robinsons de l'aprèsguerre deviennent souvent des antihéros et les frontières initiales du personnage, celles établies par Daniel Defoe, sont 
radicalement changées, Robinson devenant également un contre-Robinson.

Ce ne sont pas uniquement les frontières géographiques européennes qui sont mises en question dans la période de l'après-guerre, mais surtout une histoire et une philosophie de vie sur les plans micro et macrosocial. La métaphore de l'île inhabitée ainsi que la présence littéraire du héros moderne Robinson Crusoé ouvrent un espace propice à des fables politiques et invitent à la reconsidération des principes de l'ordre social. Comme le souligne Jean-Paul Engélibert, « depuis 1719 et la parution à Londres de Robinson Crusoé, la tradition de la robinsonnade fournit un modèle à la pensée politique » (2008, p. 183). Dans le contexte de la reconstruction sociétale européenne d'après-guerre, le système social et politique se trouve, bien entendu, au centre des préoccupations; les réécritures du mythe de Robinson connaissent une variété d'interprétations pour ses nouveaux auteurs et pour ses lecteurs. Ce personnage solitaire connaît des métamorphoses étonnantes dans différentes robinsonnades, devenant tour à tour le disciple de Vendredi, une femme, un enfant, un aliéné parmi les autres, un personnage ridicule, etc. La richesse thématique des robinsonnades offre aussi plusieurs clés de lecture et d'interprétation qui permettent au mythe de s'actualiser et de représenter des intérêts existant dans la société occidentale contemporaine :

L'homme de la rue sait qu'étant enfant il y a trouvé des histoires qui lui rappellent le camping; l'économiste en parle comme d'une fable montrant l'agent économique en prise avec la nature; le psychanalyste y repère un cas de compensation par le travail, et l'écologiste une idylle verte qui dégénère en colonie suradministrée. (Baridon, 1996, p. 69) 
Mon étude se concentre sur la reprise du mythe par Michel Tournier, intitulée Vendredi ou les limbes de Pacifique (1966), et a pour but de saisir les différences subies par ce héros solitaire dans le contexte postcolonial. Par ailleurs, je me propose d'explorer les stades et les étapes franchies par ce nouveau Robinson, qui dépasse plusieurs de ses limites et frontières intérieures et franchit des seuils construits par la civilisation occidentale; il tend notamment la main vers Vendredi, lequel représente l'inconnu, la négritude, l'orient, le différent, l'autrui, etc. Une importance particulière est attribuée aux actes de lire et d'écrire, puisque dans ce nouveau contexte mythologique, le journal de Robinson devient une radiographie de ses pensées, de ses déchirures et de sa réconciliation finale avec la solitude solaire de lîle et, encore plus important, avec soi-même.

Le plan adopté pour articuler le déplacement des frontières (sociétales, mythologiques, individuelles, etc.) dans les robinsonnades de l'après-guerre sera le suivant: tout d'abord, nous aborderons la question de la présentation de deux termes polysémiques, à savoir la frontière et l'île; puis, nous étudierons l'évolution du mythe de Robinson; et, pour finir, le rapport écriture-frontière dans Vendredi ou les limbes du Pacifique.

\section{Quelle frontière pour la frontière îlienne?}

Naufragé sur une île inconnue, Robinson Crusoé est initialement le prisonnier de cet espace, qui lui impose des frontières géographiques naturelles. Mais la relation entre Robinson et son île connaît des métamorphoses multiples, lesquelles déclenchent à chaque fois un déplacement des 
frontières, en particulier celles qui représentent une limite intérieure, liée à l'interprétation et à la subjectivité

Avant de procéder à une analyse plus détaillée du récit de Tournier, il semble essentiel de mentionner que l'île déserte, en tant qu'espace géographique et mental, semble échapper à une délimitation conceptuelle exacte. Ses frontières conceptuelles se trouvent dans un processus continu de négociation de sens et de significations. L'île elle-même facilite et crée les circonstances favorables au développement de plusieurs paradoxes qui la caractérisent : déserte, elle est toujours décrite par un observateur; elle est un lieu d'enfer et de paradis; elle constitue un espace d'utopie ou l'espace d'un non-topos.

Tout comme la notion d'île, celle de frontière possède une richesse polysémique à analyser. Le terme peut être associé à plusieurs disciplines, car la frontière peut être mise en relation avec la géographie, quand elle marque une différence naturelle, environnementale; avec l'histoire, lorsqu'elle implique une séparation conventionnelle, comme celle entre deux États; avec la science politique, aussi, puisque le sens premier du mot, qui remonte au XVI e siècle, est emprunté au domaine militaire (Soriano, 2008). La frontière est également liée au linguistique, au social, à l'urbain ou encore au juridique.

Pour Joëlle Ducos, la complexité de la frontière vient de son double sens, concret et spatial, complété par un versant symbolique et identitaire : " elle [la frontière] s'impose comme signe marqué concrètement dans le paysage, qu'il s'agisse de haies, de barrières ou des bornes, mais elle est aussi chargée symboliquement: passer la frontière, c'est parfois une transgression qui, bien au-delà d'un signe politique, peut être morale ou religieuse » $(2004$, p. 7$)$. Cette définition se retrouve 
également dans la poétique de l'espace élaborée par Gaston Bachelard, qui souligne la différence frappante entre la perception d'un espace géographique et l'expérience d'un espace métaphorique, intériorisé : «l'espace saisi par l'imagination ne peut rester l'espace indifférent livré à la mesure et à la réflexion du géomètre. Il est vécu. Et il est vécu, non pas dans sa positivité, mais avec toutes les partialités de l'imagination. » (2012, p. 17)

Cette conception de l'espace clarifie la perception de l'île déserte : celle-ci n'est pas seulement un espace physique, mais aussi un état d'esprit, une étape intérieure à appréhender, à franchir ou même à dépasser. L'imaginaire qui entoure l'île déserte ne connaît pas de limites; autrement dit, pour les îliens ou les naufragés, elle représente un espace sans frontières, puisque toutes les connotations et les interprétations symboliques sont disposées sur un axe tendu entre deux indices opposés : d'un côté, on trouve l'espace heureux, la topophilie; de l'autre, "l'espace de la haine », de la destruction (Bachelard, 2012, p.18). La pluralité de sens qui entoure l'île est une conséquence de ses caractéristiques spécifiques: en tant qu' «étendue de terre ferme émergée d'une manière durable dans les eaux d'un océan, d'une mer, d'un lac ou d'un cours d'eau » (Rey-Debove et Rey, 2013), l'île ne peut être envisagée qu'en relation avec l'immensité marine, avec le contact aquatique infini, qui ouvre, implique ou même impose la rêverie ou l'enfer sous l'effet de la solitude et l'isolement. Par ailleurs, les termes «île" et «isolement » possèdent la même racine latine, insula, et selon le même dictionnaire, les deux termes opposés à la solitude sont la compagnie et la société (ReyDebove et Rey, 2013). 
Selon Bachelard, c'est l'immensité de la mer ou de l'océan, souvent aussi l'impossibilité de quitter l'île, qui déclenchent le processus de réflexion sur le monde et sur soi : "l'immensité est en nous. Elle est attachée à une sorte d'expansion d'être que la vie refrène, que la prudence arrête, mais qui reprend dans la solitude. Dès que nous sommes immobiles, nous sommes ailleurs; nous rêvons dans un monde immense. » (2012, p. 169) Par conséquent, les personnages îliens, envahis par cet horizon marin, sont influencés et transformés par cet habitat insulaire.

Par ailleurs, la relation entre l'espace physique et l'espace mental est également analysée par Gilles Deleuze dans un essai consacré aux îles. Le philosophe propose l'origine comme caractéristique îlienne primordiale : l'île est constituée par la rencontre entre le solide et le liquide, la terre et l'océan, les principes paternel et maternel, et peut-être même par une androgynie qui suggère une origine mythique. Même si les îles ont un caractère double, géographique (réel) et mythologique (imaginaire), la dimension mythologique est beaucoup plus prégnante, en raison de son lien avec l'imaginaire collectif: «c'est dire à nouveau que l'essence de l'île déserte est imaginaire et non réelle, mythologique et non géographique » (Deleuze, 2002, p. 14). L'île est certainement un espace mythologique parce qu'elle actualise les mythes vite oubliés, mythes qui ont arrêté d'être compréhensibles dans notre société parce "qu'on ne sait plus les rêver ni les reproduire » (Deleuze, 2002, p. 14). 


\section{Sur des usages mythologiques dans la modernité tardive}

Le désenchantement de la société contemporaine occidentale et sa difficulté de vivre et de revivre les mythes expliquent en partie le sens péjoratif attaché à la notion de «mythe ». Dans le langage parlé d'aujourd'hui, le mythe est considéré comme une histoire irréelle, fausse, voire une "tromperie collective", comme l'affirme Henri Meschonnic (cité dans Brunel, 1988, p. 8).

Ce refus d'accepter le mythe au sein des pratiques sociales peut être explicable par ce que François Hartog définit comme «une crise de temps» qui caractériserait les changements de régimes d'historicité. Sur fond de rupture radicale impliquée par l'expérience de la guerre, le philosophe décrit un présent "gonflé, hypertrophié [...] où les innovations technologiques et la recherche de profits de plus en plus rapides frappent d'obsolescence les choses et les hommes de plus en plus vite» (2003, p. 125). La vitesse excessive qui caractérise la société contemporaine, son obsession du progrès et ses innovations techniques lui auraient fait perdre contact avec les mythes et les archétypes.

En même temps, la réaction à cette effervescence technologique se remarque par la contestation de l'histoire officielle, soit la valorisation de la mémoire contre l'histoire et même la recherche d'une autre histoire, conduisant à des nouvelles périodisations et explications sociétales (Hartog, 2003, p. 141). Le changement intervenu après l'expérience traumatisante de la guerre a pour conséquence une renégociation des marges concernant la place consacrée à l'individu, à l'altérité et au système social dans les 
représentations sociales contemporaines. Sur ce fond de «déchirure du monde social » (1957, p. 247), Roland Barthes consacre un ouvrage aux mythologies modernes, où il montre que les mythes existent toujours et gardent, peut-être de manière dissimulée, leur rôle de réglage et d'explication de certains faits sociaux. Pour lui, «le mythe est une parole» (Barthes, 1957, p. 193) qui transmet un message et l'organise dans un système de communication. Si on tient compte de ses usages, les significations du mythe sont ambivalentes, parfois même opposées.

Pour Michel de Certeau, le mythe de Robinson Crusoé est porteur d'une clé de lecture illustrative pour l'histoire moderne de la société occidentale, car il contient les trois éléments qui définissent la pratique historiographique moderne: «la page blanche, le texte et la construction » (1990, p. 201). Comme il le fait remarquer, le roman combine les trois traits suivants : «l'île qui découpe un lieu propre, la production d'un système d'objets par un sujet maître, et la transformation d'un monde naturel » (1990, p. 202). Les Robinsons naufragés ont toute la liberté de s'impliquer dans un processus infini d'écriture et de réécriture de leur propre histoire vécue dans l'île.

\section{Le mythe de Robinson, repère de la modernité occidentale}

Dans l'histoire des robinsonnades, une place centrale, fondatrice, est occupée par La Vie et les aventures étranges de Robinson Crusoé de York, marin de Daniel Defoe, publié en 1719 dans une Angleterre coloniale et puritaine. C'est l'ouvrage qui a " été l'objet de plus d'éditions et de traductions que tout autre livre à part la Bible» (Bevan, 1986, p. 27). Jean-Jacques 
Rousseau s'inspire également de l'histoire de Robinson pour mettre en place le projet éducatif décrit dans Émile. Selon le philosophe, Robinson constitue un modèle éducatif pour chaque garçon à l'âge de la formation.

La Vie et les aventures étranges de Robinson Crusoé de York, marin présente l'histoire d'un individu banal, enclin au vagabondage et à la fuite, qui quitte le domicile familial, fait naufrage et devient, grâce à ses expériences sur l'île, « le héros d'une histoire exceptionnelle [...] un personnage auquel chacun pourra s'identifier» (Andries, 1996, p.11). Robinson représente l'explorateur, l'aventurier, le roi de son île, le colonisateur, l'homme d'affaires, le leader politique, le père de famille, le pasteur ou le moraliste et beaucoup d'autres choses encore. Cette richesse d'interprétation transformera son histoire en mythe littéraire, puisque, dans les mots de Michel Baridon, « chacun [...] cherche ce qui l'intéresse » (1996, p. 69). Et il y a de nombreux aspects qui peuvent attirer l'intérêt dans Robinson.

Dans un premier temps, on remarque la solitude et la réaction du personnage face à l'isolement; l'organisation de la vie sur l'île; les efforts pour recréer la civilisation dans ses diverses dimensions (de la construction d'outils à la croyance); le rapport à l'altérité; le désir de liberté et de fuite, ainsi que du retour, etc. Sur un deuxième plan interprétatif, on peut concevoir Robinson comme un produit typique de sa société : c'est le petit bourgeois qui a envie de faire fortune, le fils prodigue qui quitte ses parents, le colonisateur qui impose les valeurs de l'Occident chrétien, le gouverneur de son île, infatigable dans son travail, têtu, rationnel et ordinaire. Un troisième niveau, peut-être le plus signifiant, est la frappante 
ressemblance entre l'évolution de Robinson et celle de l'histoire contemporaine: le personnage est révélateur du monde occidental, car, selon François Flahault, «Robinson existe uniquement parce qu'on en parle, et si plus personne n'en parlait, il perdrait toute existence. Robinson est un objet transindividuel, il fait partie $\mathrm{du}$ monde commun des Occidentaux, il est l'un des biens collectifs immatériels qui constituent leur culture » (2003, p. 22).

Innovante pour la fin des années 1710 est la forme du récit : le $i l$ habituel, lointain et peu impliqué dans le récit, est délaissé par Defoe au profit d'un je plus direct et proche du lecteur. Ce changement atteste de l'importance grandissante de la place de l'individu dans le milieu littéraire, mais aussi dans le milieu social. Le genre calqué sur l'autobiographie et le style réaliste font du livre le premier roman moderne d'aventures (Watt, 1996) et montrent l'intérêt pour le phénomène moderne d'individualisme, en pleine ascension.

En se basant sur la réflexion de Barthes qui affirme que l'acte « d'écrire, c'est déjà organiser le monde » (1966, p. 33), on peut concevoir deux manières successives au moyen desquelles Robinson construit son monde: la première consiste à organiser sa vie pratique de chaque jour et la seconde, à tenir un journal et à ainsi constituer un récit narratif de son existence. La préoccupation scripturale résulte de l'influence de la civilisation colonisatrice, de la Providence divine, de l'ordre tel qu'appris dans le système social d'appartenance. Robinson ne va jamais mettre en question ces discours sur le monde, il va simplement les mettre en place, les appliquer, comme si c'était plutôt sa main que son esprit qui concevait le texte du log-book. 


\section{Vendredi ou les limbes du Pacifique ou la renégociation des frontières}

Pour Tournier, Robinson est un héros des "pensées vagabondes» (Defoe, 1959, p. 17) qui a le "projet de voir le monde » (Defoe, 1959, p. 24), ce qui en fait

de tous les héros mythologiques, le plus moderne, le plus actuel. [...] Il est resté vingt ans dans l'île déserte, tout seul. Vous avez donc le problème de la solitude, et la solitude, c'est justement notre problème. Il n'y a pas de société plus dévorée, plus rongée par le drame de la solitude que la nôtre. (Tournier, 1996, p. 137)

Tout en reprenant le mythe de Robinson, Vendredi ou les limbes du Pacifique est un roman de la métamorphose qui reflète le changement du personnage et les transformations multiples de l'île. Il représente une reconfiguration évolutive de l'univers intérieur et extérieur. Les valeurs initiales sont renversées et de nouvelles conceptions sont mises en place. Le centre d'intérêt du mythe de Robinson est la métamorphose intérieure déclenchée par le voyage errant et par la rencontre avec autrui : «Robinson a assisté à la ruine des constructions sociales auxquelles il devait notamment sa sexualité de père de famille. Le voilà gratté jusqu'à l'os par ses années de solitude, libre, vierge et disponible pour les inventions les plus extravagantes » (Tournier, 1977, p.121). Compte tenu des interprétations données à son île déserte, il y a une ressemblance évidente entre Robinson et «Speranza ». Ainsi Robinson devient son île et son île devient Robinson.

À la suite de son naufrage au début du récit - comme dans le roman de Defoe - , Robinson se retrouve seul sur une île inconnue, déserte. Les barrières naturelles le forcent à y 
rester. Par la suite, il connaîtra plusieurs étapes évolutives qui impliquent chaque fois un changement de ses propres façons de percevoir le monde. La première consiste en une hypostase tellurique, quand il descend dans une cavité de Speranza et se met en position fœtale dans le corps de son île. Suit une étape végétale, celle de l'amour physique, quand Robinson assume sa maturité sexuelle et son désir de l'île-femme, devenant ainsi le père des plantes-filles, les mandragores. Ses découvertes ontologiques sont interrompues par une apparition inattendue, celle de Vendredi, sauvé par accident et dont il fait son esclave. Les relations entre les deux personnages sont déterminées par le cadre rigide imposé par le paradigme de la «mission civilisatrice ». L'explosion causée par Vendredi dans la cave aux explosifs change définitivement leur vie sur l'île et déclenche le dernier état de la métamorphose, aérien, souple, solaire. Il ne s'agit plus d'un changement des frontières, mais d'un renversement complet des valeurs, de l'ordre et des rapports humains. C'est le moment où Robinson apprend à vivre à la manière de Vendredi, où les deux commencent à jouer, à rester longtemps au soleil, à se ressembler physiquement et psychologiquement.

L'expérience îlienne et la rencontre de Vendredi que raconte Tournier relèvent d'une mise en question des grands discours de la modernité. Robinson apprend le doute : « et si en cet instant précis il découvre par hasard la beauté anatomique stupéfiante de l'œil de Vendredi, ne doit-il pas honnêtement se demander si l'Araucan n'est pas tout entier une addition de choses également admirables qu'il n'ignore que par aveuglement? » (Tournier, 1972, p. 181) 
L'île, les limites, les frontières en soi définissent et métamorphosent Robinson; sa transformation inclut une multitude d'états d'esprit, des étapes et des sentiments contradictoires. À la fin du roman, Robinson estime que sa métamorphose fut «longue et douloureuse » (Tournier, 1972, p. 226) et forcément liée à l'apparition inattendue de Vendredi. Cela reste, quand même, une décision personnelle prise dans le sens du changement. Plus exactement, il s'agit de la capacité de projeter un nouveau rêve, un nouveau projet de vie, moins contrôlé, moins ordinaire, et orienté vers une existence solaire, éternelle et atemporelle.

La transformation de Robinson n'est pas seulement psychologique, mais également physique puisque la vie sur l'île s'imprime sur son corps sous la forme de cicatrices, de brûlures, de coupures et de callosités; son existence est inscrite sur son corps, chaque cicatrice racontant une des expériences de l'île. Il $\mathrm{y}$ a également une deuxième manière, plus prégnante, d'enregistrer et d'écrire son histoire sur l'île et de prendre conscience de soi-même : c'est la décision et la joie de tenir un journal dans un des livres avec les pages blanches, lavées par l'eau. Il se consacre à cet acte de mémoire et de recollection avec beaucoup d'engagement : " il lui semblait soudain s'être à demi arraché à l'abîme de bestialité où il avait sombré et faire sa rentrée dans le monde de l'esprit en accomplissant cet acte sacré : écrire » (Tournier, 1972, p. 43). L'écriture du héros ne vise pas à tracer le temps ou à recenser les événements de ses jours, mais à consigner "ses méditations, l'évolution de sa vie intérieure, ou encore les souvenirs qui lui revenaient de son passé et les réflexions qu'ils lui inspiraient » (Tournier, 1972, p. 45). 
À la fin du roman, le bateau longtemps attendu arrive. Mais Robinson n'a plus envie de quitter son île, de redevenir comme les autres, puisque pour lui, maintenant, la civilisation qui se trouve ailleurs est superficielle et superflue. C'est Vendredi qui partira, attiré par les mécanismes sophistiqués du bateau. Un nouvel habitant remplacera ce dernier; il s'agit d'un petit garçon roux, qui fait figure d'un Robinson rajeuni.

\section{Conclusions sur le mythe de Robinson Crusoé : une écriture sans frontière?}

Pour la société occidentale de l'après-guerre, les frontières subissent un fort déplacement sur le fond d'intenses questionnements sur les plans politique, social, philosophique et économique. Les frontières qui séparaient l'acceptable et l'inacceptable, le dominant et le dominé, le moi et l'autre, le corps et l'âme, etc. sont renégociées, changées ou au moins relativisées. Les anciennes forteresses ou piliers de la pensée moderne (l'État national, l'Église, la famille, le patriotisme, etc.) ne sont plus capables de garder leur place dans l'imaginaire collectif. L'histoire elle-même est mise en question et souvent réinterprétée d'une façon différente. Toutefois, le mythe de Robinson reste représentatif pour cette nouvelle société, parce qu'il change avec elle et se débarrasse des anciennes frontières de sa conception.

Le topos de l'île est propice aux fictions sociopolitiques de par sa nature même : espace clos et supposément vierge, il peut aisément figurer le lieu de l'origine ou se présenter comme le laboratoire où il est possible de reconstituer les commencements de la société afin d'en dégager l'essence. Entre 
l'île et le monde, d'une part, et entre la petite population insulaire et l'humanité, d'autre part, il est tentant de dresser une équivalence. Les barrières géographiques que l'île impose à ses Robinsons sont fixes, immobiles, mais cette limitation physique ouvre l'espace sur l'analyse et la réflexion. Ils ont le choix de s'y soumettre ou pas.

L'envie de lire et d'écrire des récits robinsoniens est aussi alimentée par une attirance vers l'aventure, le vagabondage et l'inconnu en tant qu'ils peuvent être menés par un personnage commun, ordinaire, qui découvre et construit, petit à petit, un nouveau monde. La construction du monde n'est plus la liberté et la responsabilité des rois et des monarques; elle devient, dans la société occidentale, sinon une activité, au moins une préoccupation des gens communs. Le Robinson de Defoe est bien intégré, même fixé dans son système social et politique d'origine. Le Robinson de Tournier apprend à franchir la frontière de penser le monde imposée par l'appartenance à ce système; à faire un pas en arrière et à analyser ce monde selon ses critères personnels.

La dimension intérieure, l'odyssée en soi qui accompagne le voyage et les expériences extérieures du héros solitaire Robinson, la description de sa métamorphose, l'ordre extérieur qu'il essaye initialement d'imposer sur l'île et qu'il délaisse ultérieurement au profit d'un laisser-aller îlien sont les thèmes centraux dans la reprise du mythe réalisée par Tournier. En suivant le périple de Robinson, le lecteur prend conscience de l'histoire du colonialisme, de l'impact de la religion, du désir de mesurer et de maîtriser le temps, de la nature et de la perception d'autrui, etc. Au-delà de ces grands discours de la modernité, nous trouvons également la description de la 
recherche de soi-même que Robinson a réalisée et la manière dont il a franchi les seuils des constructions sociales: il découvre une nouvelle sexualité; il accepte Vendredi en tant qu'égal; il apprend à douter, à jouer et à faire d'autres rêves.

Son journal atteste également de son besoin d'individualisme et de son désir de liberté d'expression. Si, dans le roman de Defoe, Robinson suivait la supposée loi divine et utilisait son log-book pour marquer les faits quotidiens de sa vie îlienne, le nouveau Robinson utilise l'écriture pour garder une trace de ses sentiments et de ses méditations passés. Les deux lisent la Bible, mais le résultat est complètement différent. En fait, le premier Robinson n'écrit pas, mais plutôt inscrit sur papier ce que la Providence avait déjà tracé pour lui. Par contre, le Robinson de Tournier illustre dans ses écrits son rapport au monde et aux choses. La sortie du silence, qui s'achève sur les pages lavées par l'eau, est un acte de joie, de libération. Pas seulement parce que Robinson déplace les frontières de sa pensée en notant ses méditations et ses souvenirs, ses projets et ses nouveaux rêves, mais parce que l'acte d'écriture constitue aussi l'ancrage dans la mémoire, la création d'un patrimoine immatériel, la consignation d'une histoire personnelle qui reflète celle d'un régime d'historicité. 


\section{Bibliographie}

ANDRIES, Lise. (1996), Robinson, Paris, Autrement, coll. « Figures mythiques ».

BACHELARD, Gaston. (2012 [1981]). La Poétique de l'espace, Paris, PUF, coll. « Quadrige».

BARIDON, Michel. (1996), «Robinson, jardinier en l'île», dans Lise ANDRIEs (dir.), Robinson, Paris, Autrement, coll. « Figures mythiques», p. 69-85.

BARThES, Roland. (1957), Mythologies, Paris, Seuil, coll. « Pierres vives ».

—. (1966), Critique et vérité, Paris, Seuil, coll. « Tel quel ».

Bevan, David Gordon. (1986), Michel Tournier, Amsterdam, Rodopi, coll. «Monographique Rodopi en littérature française contemporaine».

BRUNEL, Pierre. (1988), Dictionnaire des mythes littéraires. Monaco, Éditions du Rocher.

De Certeau, Michel. (1990 [1985]), L'Invention du quotidien 1. Arts de faire, Paris, Gallimard, coll. « Folio».

DEFoE, Daniel. (1975 [1783]), Robinson Crusoé, Paris, Gallimard, coll. « Mille soleils ».

DeLEUZE, Gilles. (2002 [1993]), L'Île déserte et autres textes, 1953-1974, Paris, Minuit, coll. « Paradoxe ».

DORTIER, Jean-François. (2004), Le Dictionnaire des sciences humaines, Auxerre, Sciences Humaines Éditions. 
DuCos, Joëlle. (2004), Frontières et seuils. Cahiers du Laboratoire pluridisciplinaire de recherches sur l'imaginaire appliquées à la littérature, Bordeaux, LAPRIL-Université Bordeaux Montaigne.

ENGÉLIBERT, Jean-Paul. (2008), «L'empreinte de l'homme», Écologie \& politique, vol. 3, no 37, p.181-194, $<$ www.cairn.info/revue-ecologie-et-politique-2008-3-page181.htm>.

Flahault, François. (2003), Le Paradoxe de Robinson. Capitalisme et société, Paris, Mille et Une Nuits, coll. «Les petits libres $»$.

HARTOG, François. (2003 [2001]), Régimes d'historicité, Paris, Seuil.

Rey-Debove, Josette et Alain Rey (dir.). (2013), Le Petit Robert de la langue française, Paris, Le Robert, < https://petitrobert.aria.ehess.fr/aidepr1/Aide.htm>.

Soriano, Paul. (2008), «Frontière », Médium, vol. 2, no 15, p.180-185, <www.cairn.info/revue-medium-2008-2-page180.htm>.

TOURnIER, Michel. (1972 [1967]), Vendredi ou les limbes du Pacifique, Paris, Gallimard.

WATT, Ian. (1996), Myths of Modern Individualism: Faust, Don Quixote, Don Juan, Robinson Crusoé, Cambridge, Cambridge University Press. 


\title{
Résumé
}

Après la Seconde Guerre mondiale, les reprises littéraires du mythe de Robinson Crusoé, telle que Vendredi ou les limbes $d u$ Pacifique, sont évocatrices des changements existant dans la société européenne en reconstruction. Le laboratoire de l'espace insulaire propose un espace métaphorique de réflexion sur le système social ainsi que sur les transformations intérieures subies par les héros qui habitent l'île. Les adaptations mythologiques esquissent le passage vers un nouveau régime d'historicité, où les avatars de Robinson ont souvent dépassé les frontières conceptuelles imposées par la modernité.

\begin{abstract}
After the Second World War, the narratives of the insular space - such as Friday, or The Other Island by Michel Tournier become significant for the undergoing changes in the Western Europe. The updating of Robinson Crusoe's myth implies a continuous interest in an alternative social system and in 'otherness'. It equally reflects the transition to a new sociohistorical approach, as the new avatars of Robinson usually overpass the modern paradigm of illustrating society.
\end{abstract}

